\title{
基于人行走能耗分析的踝关节外骨骼设计
}

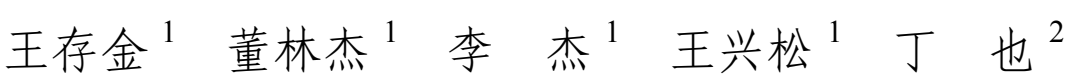 \\ (1. 东南大学机械工程学院 南京 211189; \\ 2. 远也科技(苏州)有限公司 苏州 215000)
}

\begin{abstract}
摘要: 基于对人体行走的能耗分析, 设计了一种新型有源无动力踝关节外骨骼。首先, 基于耦合摆模型建立人体行走摆动相 动力学方程并采用打靶法求解。根据动量守恒原理分别计算摆动腿滕关节锁定、脚跟着地以及关节摩擦引起的能耗率。计算 结果表明, 脚跟着地引起的能耗率远大于滕关节锁定和关节摩擦引起的能耗率。然后, 基于该能耗分析结果设计了一种足底 弹性储能机构将脚跟着地时的能耗存储起来, 在跖屈蹬地阶段释放助力。通过前脚掌压力信号控制电磁铁驱动的离合机构, 实现对助力弹簧夹紧与释放的状态切换。样机实验结果表明: 足底储能机构可以提高外骨骼的输出力矩和功率, 提升助行能 力; 外骨骼的最大输出力矩为 $19 \mathrm{~N} \cdot \mathrm{m}$; 穿戴该外骨骼行走时小腿三头肌激活度相较于不穿戴时最大下降约 $8.6 \%$ 。踝关节角 度测量结果表明，在摆动相期间外骨骼很少干扰穿戴者踝关节的正常活动。
\end{abstract}

关键词: 外骨骼; 踝关节; 离合机构; 能量回收; 耦合摆

中图分类号: TP24

\section{Design of Ankle Exoskeleton Based on Analysis on Energy Cost of Human Walking}

\author{
WANG Cunjin $^{1}$ DONG Linjie ${ }^{1} \quad$ LI Jie $^{1} \quad$ WANG Xingsong $^{1} \quad$ DING Ye $^{2}$ \\ (1. School of Mechanical Engineering, Southeast University, Nanjing 211189; \\ 2. Yrobot (Suzhou) Co., Ltd., Suzhou 215000)
}

\begin{abstract}
A new type of active unpowered ankle exoskeleton is designed based on the analysis on the energy cost of human walking. Firstly, dynamical equations of swing phase of human walking are built based on a coupled pendulum model and shooting method is used to solve the equations. Energy cost rate of knee-lock, heel-strike and joint friction of the swing leg are calculated based on the principle of momentum conservation. The calculation results show that the energy cost rate of heel-strike is much greater than the energy cost rate resulted from knee-lock and joint friction. Then, based on the results of analysis of the energy cost, an plantar elastic storage mechanism is designed to store the energy cost of heel-strike, which is released during the plantar flexion push-off phase. Pressure signals from forefoot are used to control the clutch mechanism driven by electromagnet, the clamping and releasing states switching of assistive spring is achieved. Experimental results of the prototype shown that plantar energy storage mechanism can increase the output torque and power of the ankle exoskeleton, enhance the ability of assisting walking. The maximum output torque is $19 \mathrm{Nm}$. The muscle activity of the triceps surae is reduced by $8.6 \%$ when wearing the exoskeleton compared with the state without wearing the exoskeleton. The measurement results of ankle joint angle show that the exoskeleton rarely interferes with the normal range of motion of the wearer's ankle during swing phase.
\end{abstract}

Key words: exoskeleton; ankle joint; clutch mechanism; energy recycle; coupled pendulum

\section{0 前言}

行走是人类日常运动的主要形式, 据统计现代 人平均每天行走约一万步, 一生中行走约上亿步 ${ }^{[1]}$ 。 人体的双足行走是一项看似简单实则非常复杂的运 动, 众多肌肉群 ${ }^{[2]}$ 、骨骼 ${ }^{[3]}$ 和神经组织 ${ }^{[4]}$ 都参与其中。

* 江苏省研究生科研创新计划资助项目(KYCX17_0060)。20201107 收到初 稿, 20210517 收到修改稿
经过数百万年的进化, 人体生长出(或退化掉)相应 的肌肉与骨骼组织, 已经非常精于行走, 可进行长 达数天甚至数周的行走运动, 更能在几分钟甚至几 秒内适应新的行走环境 ${ }^{[5]}$ 。

踝关节作为人体与地面接触的枢纽, 其功能的 好坏直接影响人行走的稳定性和持续性 ${ }^{[6]}$ 。在行走 中的一些关键时刻, 如足部蹬地、支撑体重以及摆 动腿启动等, 踝关节均起到了重要作用。踝关节在 人行走期间输出的正功率相较于同侧的髋关节和膝 
关节更是达到了总正功率的 $46 \%{ }^{[7]}$ 。

基于踝关节在人行走过程中的重要作用，许多 外骨骼研究团队已将注意力集中于踝关节。现有的 踝关节外骨骼大致可分为三类。第一类是动力外骨 骼, 典型代表是美国麻省理工学院 HERR 团队 ${ }^{[8]}$ 的 踝关节动力外骨骼, 该外骨骼通过一个固定在小腿 处的电动绞盘机, 牵拉一对固定在靴子上的玻璃纤 维杆, 在踝关节跖屈阶段提供助力。卡内基 - 梅隆 大学 ZHANG 等 ${ }^{[9]}$ 提出一种采用电机套索驱动的踝 关节动力外骨骼, 可根据穿戴者的能耗特点采用进 化策略对外骨骼的输出力矩实时优化以达到降低穿 戴者行走能耗的目的。由于驱动电机、控制系统和 电源等硬件均外置于专门的平台上而非由穿戴者携 带, 因此该外骨骼系统的实用性受限。第二类是无 动力外骨骼, 典型的代表是卡内基·梅隆大学 COLLINS 等 ${ }^{[10]}$ 开发的踝关节无动力外骨骼, 该外骨 骼的纯机械棘轮离合机构由穿戴者的踝关节角度触 发状态切换, 控制小腿后侧助力弹簧的拉伸储能与 收缩释能, 实现降低踝关节跖屈力矩与小腿肌肉群 激活度的目的。该款外骨骼表明, 人体臻于完美的 行走能力仍有提升空间, 且外部能量输入并非外骨 骼助力的必要条件。该外骨骼的局限性在于纯机械 离合机构的状态切换有赖于穿戴者水平匀速行走时 特定的踝关节角度, 若该特定角度发生变化(如替换 不同穿戴者)容易导致离合机构触发失败 ${ }^{[1]}$ 。为解决 纯机械外骨骼适应能力差的问题, 该团队 ${ }^{[12]}$ 又提出 第三类外骨骼, 即有源无动力外骨骼。该外骨骼采 用电粘离合器替代机械离合器, 该离合器基于涂有 介电材料的薄电极片之间的静电粘着特性设计。橡 胶弹簧与离合器串联, 通过调节并联橡胶弹簧的数 量控制总刚度系数。由于引入了传感与控制系统, 该外骨骼对不同穿戴者的适应能力强于无动力外骨 骼, 且能耗远小于动力外骨骼。由于运行时需要用 到 $240 \mathrm{~V}$ 的电压, 存在一定的安全风险。

相比于国外, 国内对踝关节外骨骼的研究起步 较晚。代表研究有北京交通大学郭盛团队 ${ }^{[13]}$ 研制的 采用纯机械式传感机构的无动力踝关节外骨骼, 可根 据足底与地面的接触状态控制与储能弹簧相连的离合

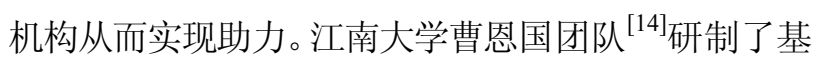
于多级能量锁原理的被动式外骨骼机械足, 通过一 个带有弹簧的可穿戴被动式纯机械装置实现能量回 收。北京航空航天大学 YANG 等 ${ }^{[15]}$ 设计了一款柔性 索驱动的动力踝关节外骨骼, 用一个电机驱动跖屈 与背屈两个方向的关节运动。南方科技大学 $\mathrm{FU}$ 等 ${ }^{[16]}$ 设计了一款半被动下肢外骨骼, 其离合机构采用低能
耗的微型伺服电机驱动, 实现了将膝关节在摆动相末 期所做负功进行存储并向同侧踝关节转移的功能。

针对动力外骨骼需要携带较大质量的驱动器和 电源, 而无动力外骨骼无法自动适应穿戴者步态变 化的问题 ${ }^{[17]}$, 本研究基于人体行走能耗分析, 设计 了一款有源无动力踝关节外骨骼, 将脚跟着地能耗 存储于足底弹性储能机构, 并在跖屈蹬地阶段释放 助力。该外骨骼采用了一种由前脚掌足底压力信号 控制的离合机构, 避免了纯机械离合机构因触发角 度变化而导致的状态切换失败。所耗电能仅用于离 合机构的驱动和传感与控制系统。最后, 开展行走 实验验证该踝关节外骨骼的有效性。

\section{1 人体行走模型}

\section{1 摆动相动力学模型}

人体行走过程中, 单侧下肢的运动状态可划分 为支撑相与摆动相。支撑相始于脚跟着地 (Heel-strike), 止于脚尖离地(Toe-off), 约占整个步 态周期的 $60 \%$; 摆动相始于脚尖离地, 止于脚跟着 地, 约占整个步态周期的 40\%。由于行走的对称性, 整个步态周期又可分为双支撑相和单支撑相, 分别 占步态周期的 $20 \%$ 和 $80 \%$ 。建立人体水平行走单侧 腿的摆动相模型如图 1 所示。该模型是位于人体矢 状面内的二维平面模型, 包括 5 个连杆: 两个脚部 杆、支撑腿杆、大腿杆和小腿杆。其中, 一个脚部 杆固连于地面, 踝关节近似为一个铰链将该脚部杆 与支撑腿杆相连, 此时支撑腿杆可看作是一个倒立 摆 ${ }^{[18]}$ 。另一个脚部杆和小腿杆的一端垂直固连, 小 腿杆的另一端和大腿杆铰接, 构成摆动腿。摆动腿 在髋关节处和支撑腿杆铰接。根据以上连接假设, 该五杆模型实质为三杆模型。图 1 中各符号的含义 为: $L_{l} 、 Z_{l} 、 M_{l} 、 \theta 、 \dot{\theta}$ 分别是支撑腿的长度、质 心距离、质量、与坚直线所成的角度与角速度, $L_{t}$ 、 $Z_{t} 、 M_{t} 、 \varphi 、 \dot{\varphi}$ 分别是摆动腿大腿的长度、质心距 离、质量、与坚直线所成的角度与角速度, $L_{s} 、 Z_{s}$ 、 $M_{s} 、 \sigma 、 \dot{\sigma}$ 分别是摆动腿小腿的长度、质心距离、 质量、与坚直线所成的角度与角速度, $M_{u} 、 M_{T}$ 分别 是上身的质量与身体的总质量, $d$ 为足长, $S_{L}$ 步长, $\alpha$ 为脚与地面所成角度。另外，规定逆时针方向为正。 采用 Dempster 的人体测量学数据赋予该耦合摆模 型人体测量学参数 ${ }^{[19]}$, 以上变量有如下数学关系 $L_{l}=L_{t}+L_{s}, L_{t}=L_{s}, Z_{t} / L_{t}=0.433, Z_{s} / L_{s}=0.437$, $Z_{l} M_{l}=Z_{t} M_{t}+\left(Z_{s}+L_{t}\right) M_{s}, \quad M_{l}=M_{t}+M_{s}$, $M_{t} / M_{T}=0.097, M_{s} / M_{T}=0.06, d=L_{l} / 4$ 。 


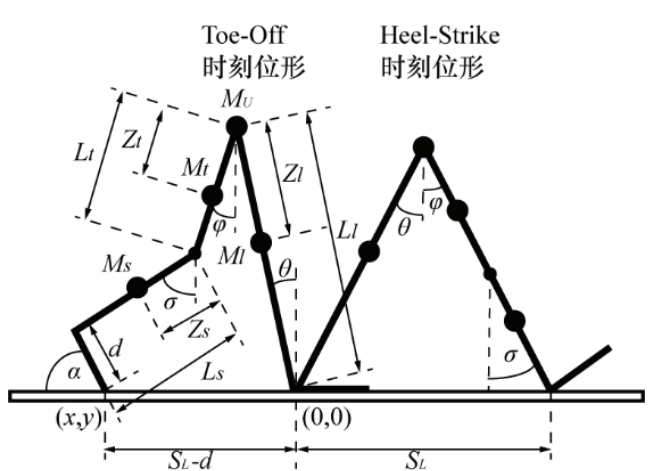

图 1 人体行走摆动相模型

该耦合摆模型有 3 个自由度, 其广义坐标可表 示为 $(\theta, \varphi, \sigma)$, 该系统的动能和势能分别为

$$
\begin{aligned}
K E & =\frac{1}{2} \sum_{i, j=1}^{3} c_{i j} \dot{\phi}_{i} \dot{\phi}_{j} \\
P E & =\sum_{i=1}^{3} w_{i} \cos \phi_{i}
\end{aligned}
$$

式 中 , $c_{i j}=\bar{c}_{i j} \cos \left(\phi_{i}-\phi_{j}\right), i, j=1,2,3$ 且 $\left(\phi_{1}, \phi_{2}, \phi_{3}\right)=(\theta, \varphi, \sigma) \cdot\left(\bar{c}_{11}, \bar{c}_{22}, \bar{c}_{33}\right)=\left(M_{T} L_{l}^{2}-2 M_{l} L_{l} Z_{l}+\right.$ $\left.M_{l} Z_{l}^{2}, M_{t} Z_{t}^{2}+M_{s} L_{t}^{2}, M_{s} Z_{s}^{2}\right),\left(\bar{c}_{12}, \bar{c}_{13}, \bar{c}_{23}\right)=\left(-M_{t} L_{l} Z_{t}-\right.$ $\left.M_{s} L_{l} L_{t},-M_{s} L_{l} Z_{s}, M_{s} L_{t} Z_{s}\right),\left(\bar{c}_{21}, \bar{c}_{31}, \bar{c}_{32}\right)=\left(-M_{t} L_{l} Z_{t}-\right.$ $\left.M_{s} L_{l} L_{t},-M_{s} L_{l} Z_{s}, M_{s} L_{t} Z_{s}\right),\left(w_{1}, w_{2}, w_{3}\right)=\left(M_{T} L_{l} g-\right.$ $\left.M_{l} Z_{l} g,-M_{t} Z_{t} g-M_{s} L_{t} g,-M_{s} Z_{s} g\right)$ 。

耦合摆系统的总能量可表示为

$$
E=P E+K E
$$

人在行走过程中受到的保守力为重力, 非保守 力主要包括肌肉力和关节摩擦力。根据肌电信号分 析, 当人体以自然舒适的步速行走时, 摆动腿的肌 肉几近静息, 即几乎不输出肌肉力 ${ }^{[20]}$, 因此可认为 在摆动相期间的非保守力只有关节摩擦力。当考虑 关节摩擦并假定关节摩擦力矩与关节旋转速度成正 比时，描述摆动相的动力学微分方程组为

$$
\begin{gathered}
{\left[\begin{array}{lll}
c_{11} & c_{12} & c_{13} \\
c_{21} & c_{22} & c_{23} \\
c_{31} & c_{23} & c_{33}
\end{array}\right]\left[\begin{array}{c}
\ddot{\theta} \\
\ddot{\varphi} \\
\ddot{\sigma}
\end{array}\right]+\left[\begin{array}{ccc}
0 & c_{12}^{*} & c_{13}^{*} \\
c_{21}^{*} & 0 & c_{23}^{*} \\
c_{31}^{*} & c_{32}^{*} & 0
\end{array}\right]\left[\begin{array}{l}
\dot{\theta}^{2} \\
\dot{\varphi}^{2} \\
\dot{\sigma}^{2}
\end{array}\right]=\left[\begin{array}{l}
w_{1} \sin \theta \\
w_{2} \sin \varphi \\
w_{3} \sin \sigma
\end{array}\right]+} \\
{\left[\begin{array}{ccc}
-b_{1}-b_{2} & b_{2} & 0 \\
b_{2} & -b_{2}-b_{3} & b_{3} \\
0 & b_{3} & -b_{3}
\end{array}\right]\left[\begin{array}{c}
\dot{\theta} \\
\dot{\varphi} \\
\dot{\sigma}
\end{array}\right]}
\end{gathered}
$$

式中, $b_{1} 、 b_{2} 、 b_{3}$ 分别为支撑腿的踝关节、摆动腿 的髋关节与膝关节的粘滞摩擦系数。

由于摆动相初始时刻下肢各关节的角速度未 知, 式(4)无法构成初值问题, 可通过预设摆动相起 止时刻各关节的角度值, 将求解该方程组变为求解 一个两点边界值问题。采用打靶法求解该边界值问
题, 每次 “打靶” 使用 4 阶龙格-库塔法计算。当 $b_{1}=0.5 、 b_{2}=0.001 、 b_{3}=0.015$ 时 ${ }^{[19]}$, 根据表 1 中的 初始时刻条件, 采用打靶法可得出三关节在摆动时 间 $T=0.4 \mathrm{~s}$ 内的角度变化, 如图 3 所示。各关节起 止时刻的角速度如表 2 所示。

表 1 摆动相起止位置各关节的角度

\begin{tabular}{lcc}
\hline & 初始时刻(脚尖离地) & 终止时刻(脚跟着地) \\
\hline$\theta /\left(^{\circ}\right)$ & 10 & -22.02 \\
$\varphi /\left(^{\circ}\right)$ & -9.4 & 22.02 \\
$\sigma /\left(^{\circ}\right)$ & -55 & 22.02 \\
\hline
\end{tabular}

表 2 摆动相起止时刻各关节的角速度

\begin{tabular}{lcc}
\hline & 初始时刻(脚尖离地) & 终止时刻(脚跟着地) \\
\hline$\theta /(\mathrm{rad} / \mathrm{s})$ & -1.47 & -1.69 \\
$\varphi /(\mathrm{rad} / \mathrm{s})$ & 3.9 & 0.44 \\
$\sigma /(\mathrm{rad} / \mathrm{s})$ & -5.47 & 1.73 \\
\hline
\end{tabular}

为验证耦合摆模型摆动相下肢各关节角度理论 值的准确性，使用人体运动捕捉系统测量人体行走 运动学数据, 并将以上理论计算值与实测值进行比 较。实验现场如图 2 所示, 测试者身高 $177 \mathrm{~cm}$, 体 重 $70 \mathrm{~kg}$, 以自然舒适的步速(约 $1.2 \mathrm{~m} / \mathrm{s}$ )进行行走测 试。根据发光标记点的位置坐标计算出人体行走过 程中下肢各关节的角度变化曲线，如图 3 所示。由 图 3 可知, 理论计算的支撑腿踝关节角度 $\theta$ 和摆动 腿髋关节角度 $\varphi$ 接近人体行走时的实际角度。摆动 腿膝关节最大屈曲角度 $\sigma=61.41^{\circ}$, 接近人体行走时 膝关节最大屈曲角度 $\left(\right.$ 约 $\left.60^{\circ}\right)$ 。

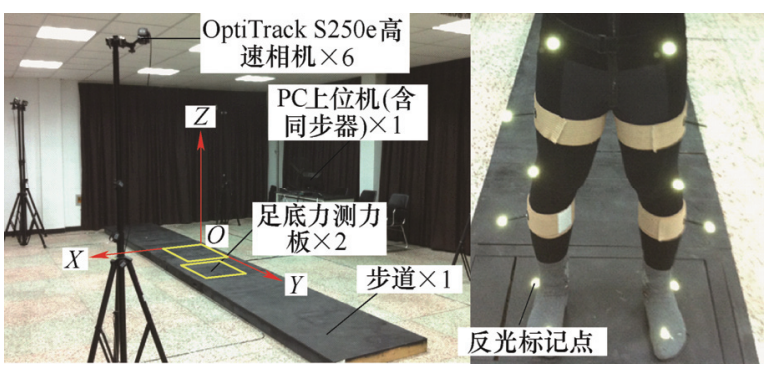

图 2 人体运动捕捉系统

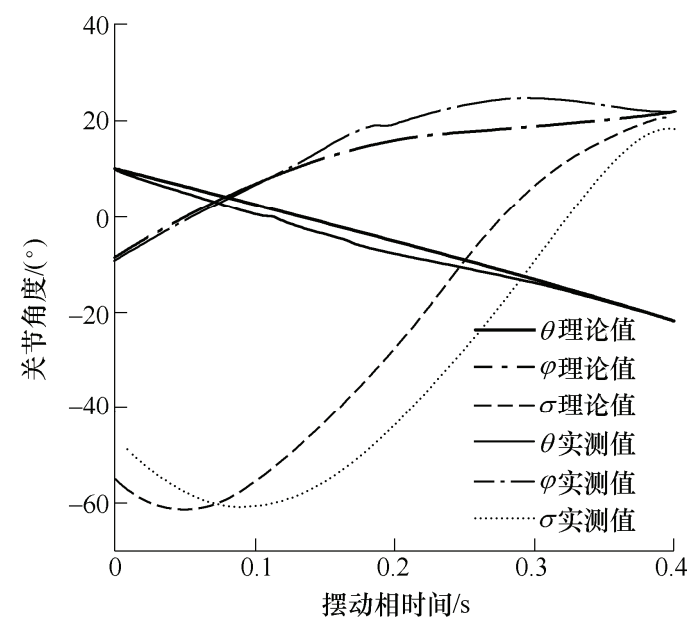

图 3 摆动相各关节角度理论计算值与实测值 


\section{2 双支撑相运动学模型}

在双支撑期间, 摆动腿和支撑腿切换角色，原 摆动腿变为新支撑腿, 原支撑腿变为新摆动腿。由 于双支撑期间原支撑腿的脚尖与地面保持接触且无 滑移, 可近似为与地面铰接。若将摆动腿的脚跟与 地面接触的点作为下一步的原点(图 1), 则在脚跟着 地的 $T$ 时刻的坐标变为 $(\bar{\theta}, \bar{\varphi}, \bar{\sigma}, \bar{\alpha})$, 其 中 $\bar{\theta}(T)=\sigma(T), \quad \bar{\varphi}(T)=\theta(T) \quad, \quad \bar{\sigma}(T)=\theta(T)$, $\bar{\alpha}(T)=0$ 。在双支撑相期间, 原支撑腿的脚尖 $(x, y)$ 可表示为 $\left\{\begin{array}{l}x(t)=d-S_{L}=-L_{l} \sin \bar{\theta}+L_{t} \sin \bar{\varphi}+L_{s} \sin \bar{\sigma}+d \cos \bar{\alpha}, \\ y(t)=0=L_{l} \cos \bar{\theta}-L_{t} \cos \bar{\varphi}-L_{s} \cos \bar{\sigma}+d \sin \bar{\alpha} \cdot \bar{\alpha},\end{array}\right.$

由于双支撑相期间脚尖与地面保持相对静止, 则该坐标对时间的导数 $\left(x_{t}^{\prime}, y_{t}^{\prime}\right)=0$, 即 $\left\{\begin{array}{l}x^{\prime}(t)=0=-L_{l} \cos \bar{\theta} \cdot \bar{\theta}+L_{t} \cos \bar{\varphi} \cdot \bar{\varphi}+L_{s} \cos \bar{\sigma} \cdot \bar{\sigma}- \\ d \sin \bar{\alpha} \cdot \bar{\alpha} \\ y^{\prime}(t)=0=-L_{l} \sin \bar{\theta} \cdot \bar{\theta}+L_{t} \sin \bar{\varphi} \cdot \bar{\varphi}+L_{s} \sin \bar{\sigma} \cdot \bar{\sigma}+ \\ d \cos \bar{\alpha} \cdot \bar{\alpha}\end{array}\right.$

式中, $t>T$, 表示在脚跟着地之后。式(5)为双支撑 相期间耦合摆的运动学模型。

\section{2 能量损耗}

在建立耦合摆模型时假设肌肉做功的时间集中 于双支撑相期间, 则摆动相期间的能耗主要由关节 粘滞摩擦力矩、Knee-lock 和 Heel-strike 的碰撞产生。 两次碰撞的特点是在极短的时间内, 产生了较大的 瞬时碰撞力。由于该碰撞力急剧变化, 因此本研究 只分析碰撞前后肢体运动的变化并对碰撞过程做出 如下假设: (1) 碰撞瞬时完成, Knee-lock 后的膝关 节保持锁定状态, Heel-strike 后脚跟与地面无弹起 和滑移; (2) 碰撞前后耦合摆系统各关节的角度没有 发生变化, 但关节角速度发生了突变。基于以上假 设, 分别对 Knee-lock 和 Heel-strike 的能耗率展开 分析。

\subsection{Knee-lock 能耗}

膝关节锁定 (Knee-lock) 是防止摆动腿膝关节过 度伸展的约束条件。假设该碰撞为完全非弹性碰撞, 发生于 $t=T_{k}$ 时刻, 此时 $\varphi\left(T_{k}\right)=\sigma\left(T_{k}\right)$, 且 $\dot{\varphi}\left(T_{k}\right)<\dot{\sigma}\left(T_{k}\right)$ 。在锁定完成后的 $t=T_{k}^{+}$时刻, $\dot{\varphi}\left(T_{k}^{+}\right)=\dot{\sigma}\left(T_{k}^{+}\right)$, 在此过程中整个系统分别在沿着
平行和垂直于地面的方向上动量守恒。设 $(\bar{\theta}, \bar{\varphi}, \bar{\sigma}, \dot{\bar{\theta}}, \dot{\bar{\varphi}}, \dot{\bar{\sigma}})$ 为 Knee-lock 后各关节的角度和角 速度, 由 Knee-lock 前后 $x$ 方向(与地面平行)上的动 量守恒可得

$M_{x}=\left(-M_{T} L_{l}+M_{l} Z_{l}\right) \cos \theta \cdot \dot{\theta}+\left(M_{t} Z_{t}+M_{s} L_{s}\right) \cos \varphi \cdot \dot{\varphi}+$ $M_{s} Z_{s} \cos \sigma \cdot \dot{\sigma}=\left(-M_{T} L_{l}+M_{l} Z_{l}\right) \cos \bar{\theta} \cdot \dot{\bar{\theta}}+$

$\left(M_{t} Z_{t}+M_{s} L_{t}\right) \cos \bar{\varphi} \cdot \dot{\bar{\varphi}}+M_{s} Z_{s} \cos \bar{\sigma} \cdot \dot{\bar{\sigma}}$

同理，由在 $y$ 方向(与地面垂直)上的动量守恒可得 $M_{y}=\left(-M_{T} L_{l}+M_{l} Z_{l}\right) \sin \theta \cdot \dot{\theta}+\left(M_{t} Z_{t}+M_{s} L_{t}\right) \sin \varphi \cdot \dot{\varphi}+$ $M_{s} Z_{s} \sin \sigma \cdot \dot{\sigma}=\left(-M_{T} L_{l}+M_{l} Z_{l}\right) \sin \bar{\theta} \cdot \dot{\bar{\theta}}+$ $\left(M_{t} Z_{t}+M_{s} L_{t}\right) \sin \bar{\varphi} \cdot \dot{\bar{\varphi}}+M_{s} Z_{s} \sin \bar{\sigma} \cdot \dot{\bar{\sigma}}$

此外, Knee-lock 前后已知的角度变量关系是

$$
\bar{\theta}=\theta \quad \bar{\varphi}=\varphi \quad \bar{\sigma}=\sigma \quad \bar{\varphi}=\bar{\sigma} \quad \dot{\bar{\varphi}}=\dot{\bar{\sigma}}
$$

则根据式(6)与式(7)可得

$$
\begin{gathered}
\dot{\bar{\theta}}=\frac{d p-b q}{a d-b c} \quad \dot{\bar{\varphi}}=\dot{\bar{\sigma}}=\frac{a q-c p}{a d-b c} \\
a=\left(-M_{T} L_{l}+M_{l} Z_{l}\right) \cos \bar{\theta} \\
b=\left(M_{t} Z_{t}+M_{s} L_{t}+M_{s} Z_{s}\right) \cos \bar{\varphi} \\
c=\left(-M_{T} L_{l}+M_{l} Z_{l}\right) \sin \bar{\theta}
\end{gathered}
$$

$p=\left(-M_{T} L_{l}+M_{l} Z_{l}\right) \cos \theta \cdot \dot{\theta}+\left(M_{t} Z_{t}+M_{s} L_{t}\right) \cos \varphi \cdot \dot{\varphi}+$ $M_{s} Z_{s} \cos \sigma \cdot \dot{\sigma}$

$q=\left(-M_{T} L_{l}+M_{l} Z_{l}\right) \sin \theta \cdot \dot{\theta}+\left(M_{t} Z_{t}+M_{s} L_{t}\right) \sin \varphi \cdot \dot{\varphi}+$ $M_{s} Z_{s} \sin \sigma \cdot \dot{\sigma}$

经计算 Knee-lock 后关节角速度 $(\dot{\bar{\theta}}, \dot{\bar{\varphi}}, \dot{\bar{\sigma}})=(-1.7,0.7,0.7)$, 单位 $\mathrm{rad} / \mathrm{s}$ 。该碰撞前的 角速度值为摆动相末期的各关节角速度值(表 2)。将 Knee-lock 前后关节角度和角速度值代入式(1) (3) 即可得到 Knee-lock 前后的总能量 $E\left(T_{k}^{-}\right)$和 $E\left(T_{k}^{+}\right)$, 进而得到 Knee-lock 能耗率 为 $\eta=\left[E\left(T_{k}^{-}\right)-E\left(T_{k}^{+}\right)\right] / E_{0}$, 其中 $E_{0}$ 是初始时刻系统 的总机械能, 可根据式(1) (3)和初始角度值和角速 度值 (表 1 和表 2)算出。最终计算得到耦合摆模型 Knee-lock 能耗率为 $0.024 \%$ 。

\subsection{Heel-strike 能耗}

双支撑相运动学模型式(5)可改写为

$$
\begin{gathered}
\left\{\begin{array}{l}
\boldsymbol{v}_{1} \cdot \boldsymbol{V}=0 \\
\boldsymbol{v}_{2} \cdot \boldsymbol{V}=0
\end{array}\right. \\
\boldsymbol{V}=(\dot{\bar{\theta}}, \dot{\bar{\varphi}}, \dot{\bar{\sigma}}, \dot{\bar{\alpha}})
\end{gathered}
$$




$$
\begin{gathered}
\boldsymbol{v}_{1}=\left(-L_{l} \cos \bar{\theta}, L_{t} \cos \bar{\varphi}, L_{s} \cos \bar{\sigma},-d \sin \bar{\alpha}\right) \\
\boldsymbol{v}_{2}=\left(-L_{l} \sin \bar{\theta}, L_{t} \sin \bar{\varphi}, L_{s} \sin \bar{\sigma}, d \cos \bar{\alpha}\right)
\end{gathered}
$$

式(8)可理解为在整个双支撑相期间, 广义速度矢量 $\boldsymbol{V}$ 始终在一个与矢量 $\boldsymbol{v}_{1}$ 和 $\boldsymbol{v}_{2}$ 垂直的 “平面” $S$ 内。 在摆动相末期的 $t=T^{-}$时刻, $\boldsymbol{V}\left(T^{-}\right)$并不在 $\mathrm{S}$ 内, 而 Heel-strike 的作用就是将 $\boldsymbol{V}\left(T^{-}\right)$投影到 $\mathrm{S}$ 内, 得 到新的广义速度以满足式(8)的约束。新广义速度 $\boldsymbol{V}\left(T^{+}\right)$计算如下

$$
\begin{gathered}
\boldsymbol{V}\left(T^{+}\right)=\boldsymbol{V}\left(T^{-}\right)-\left(\boldsymbol{u}_{1} \cdot \boldsymbol{V}\left(T^{-}\right)\right) \boldsymbol{u}_{1}-\left(\boldsymbol{u}_{2} \cdot \boldsymbol{V}\left(T^{-}\right)\right) \boldsymbol{u}_{2} \\
\boldsymbol{u}_{1}=\frac{\boldsymbol{v}_{1}\left(T^{-}\right)}{\left\|\boldsymbol{v}_{1}\left(T^{-}\right)\right\|} \quad \boldsymbol{u}_{2}=\frac{\boldsymbol{e}}{\|\boldsymbol{e}\|} \\
\boldsymbol{e}=\boldsymbol{v}_{2}\left(T^{-}\right)-\left(\boldsymbol{u}_{1} \cdot \boldsymbol{v}_{2}\left(T^{-}\right)\right) \boldsymbol{u}_{1}
\end{gathered}
$$

经计算, Heel-strike 后各关节角速度 $(\dot{\bar{\theta}}, \dot{\bar{\varphi}}, \dot{\bar{\sigma}})=(-0.22,-0.22,-0.22)$, 单位 $\mathrm{rad} / \mathrm{s}$ 。

Heel-strike 前各关节角速度值为 Knee-lock 后的关节 角速度。在得到 Heel-strike 后的各关节角速度后, 采用与第 2.1 节相同的方法计算出 Heel-strike 的能 耗率为 $13.71 \%$ 。摩擦引起的能耗率为摆动相起始时 刻的机械能之差与 $E_{0}$ 的比值。将两次碰撞和摩擦引 起的能耗率汇总于图 4, 可知 Heel-strike 的能耗率 远大于其他能耗率。

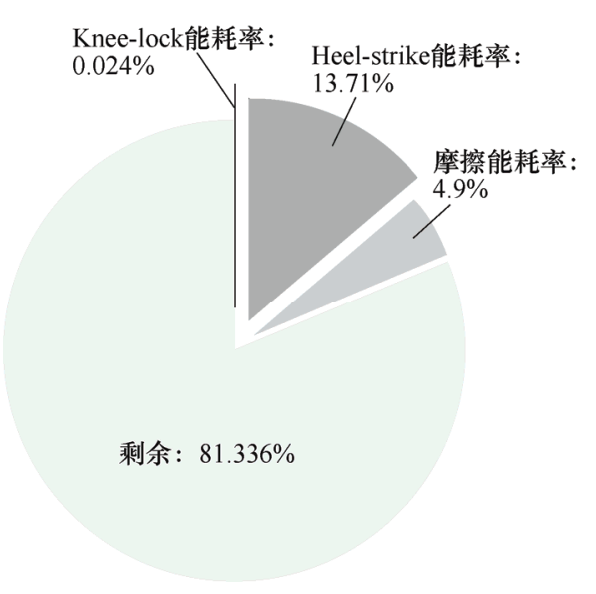

图 4 能量损耗分布

\section{3 踝关节外骨骼结构设计}

基于以上对人体行走能耗的定量分析, 可将 Knee-lock 和 Heel-strike 耗散的能量存储起来, 在人 行走关键时刻释放助力。由于 Heel-strike 的撞击能 耗远大于 Knee-lock, 因此回收 Heel-strike 的耗能更 有价值。此外, 考虑到踝关节处的小腿三头肌-跟腱
系统在蹬地时刻输出了行走时下肢各关节中最大 的正功率 ${ }^{[7]}$, 选择将踝关节作为助力的目标关节, 提出一种踝关节助力外骨骼实现能量回收: 在脚 跟着地阶段, 地面反作用力拉伸外骨骼足底的储 能弹簧, 利用弹簧为下肢提供缓冲, 将人体的部 分动能和重力势能转化为弹性势能。在支撑相末 期, 储存的弹性势能被释放, 为踝关节肌肉群提 供助力。

踝关节外骨骼的机构总重 $850 \mathrm{~g}$, 包括位于足部 的弹性储能机构和位于小腿背部的串联弹性储能机 构(图 5), 以下分别作详细介绍。

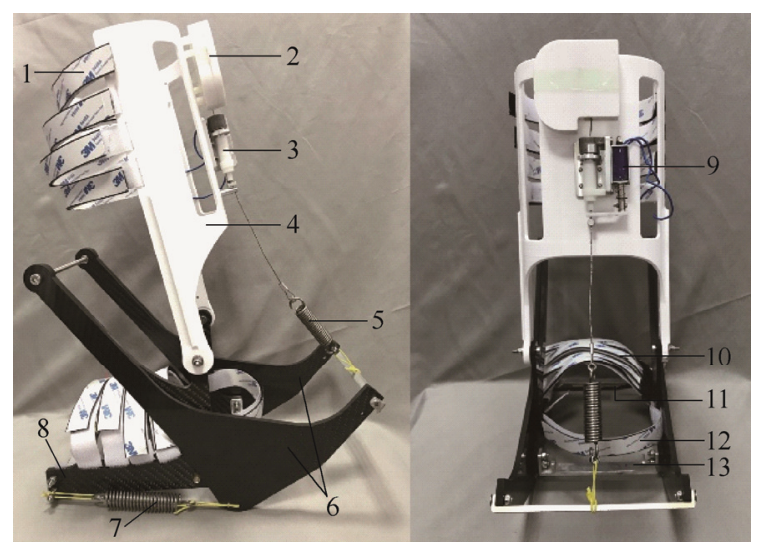

图 5 踝关节外骨骼整体结构图
1. 小腿绑带 2. 收线机构 3. 可控离合机构 4. 小腿板
5. 串联弹簧 6. 两侧板 7. 足底储能弹簧 8. 足底框架
9. 推拉式电磁铁 10. 脚背绑带 11. 前脚板 12. 脚跟绑带

13. 后脚板

\section{1 足部弹性储能机构设计}

足部弹性储能机构主要由足底框架、两侧板、 足底储能弹簧、脚背和脚跟绑带组成。足底框架的 前后脚板为铝合金材质, 其余部分和两侧板为碳纤 维材质。足底框架、两侧板和弹簧构成一个三角形 的弹性储能机构(图 6)。

该机构有两种状态: (1) 初始状态; (2) 储能状 态。在(1)初始状态, 两侧储能弹簧对侧板施加一定 的初拉力, 合力为 $F_{1}$, 而两侧板又被位于足底框架 $\mathrm{A}$ 位置的限位销挡住, 以维持机构的稳定。在(2)储 能状态, 足底框架完全着地, 两侧板的下边缘与地 面相切, 两侧弹簧拉伸储能。从状态(1)到状态(2), 对应了人行走时从脚跟刚着地到脚掌放平的 Heel-strike 过程。为保证在该过程中储能弹簧顺利 拉伸, 需要在状态(2)时, 两侧弹簧最大拉伸合力 $F_{2}$ 满足

$$
F_{2} D_{2}<N D_{1}
$$

式中, $N$ 为作用在两侧板与地面切点处的地面反 
作用力, $D_{1}$ 和 $D_{2}$ 分别为两侧板旋转轴到 $N$ 和 $F_{2}$ 的作用线的垂直距离, 分别为 $169.8 \mathrm{~mm}$ 和 $188.9 \mathrm{~mm}$ 。实测测试者(体重约 $70 \mathrm{~kg}$ ) 以三种步速行 走时脚跟处的足底力, 根据测试者一个步态周期内 脚跟处足底力的变化情况(图 7), 若 $N$ 取值为 $450 \mathrm{~N}$, 此时, $F_{2}$ 应小于 $404.5 \mathrm{~N}$, 单侧弹簧拉力应小于 202.3 $\mathrm{N}$ 。在选择两侧弹簧刚度系数时, 若弹簧的刚度系 数较大, 人体会受到较大的斜后方力, 可能将人体 弹回; 若弹簧的刚度系数较小, 则装置储存的能量 较少, 在蹬地时只能提供很少的能量。在状态(1), 两侧弹簧拉力的合力经调节为 $F_{1}=248 \mathrm{~N}$ 时, 考虑到 弹簧的最大拉伸量为 $\Delta d=d_{2}-d_{1}=22.1 \mathrm{~mm}$, 该外骨骼 选择的足底储能弹簧刚度系数为 $K=3 \mathrm{~N} / \mathrm{mm}$, 此时 $F_{2}=380.5 \mathrm{~N}$, 满足式(10)。

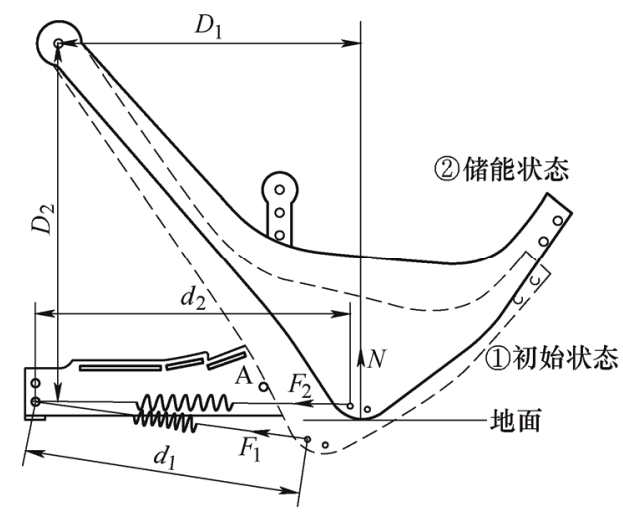

图 6 足底弹性储能机构着地过程

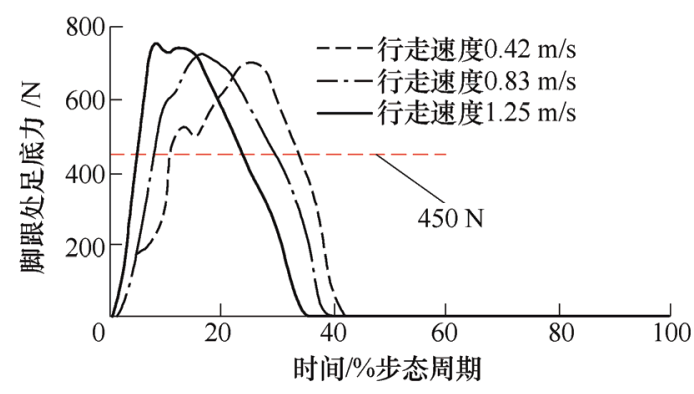

图 7 脚跟足底力在步态周期内的变化

\section{2 串联弹性储能机构}

小腿背部的串联弹性储能机构主要包括: 收线 机构、可控离合机构、串联弹簧、小腿绑带(图 5)。 收线机构(图 8a)包括外壳、平面卷簧和收线轮, 绳 索(直径 $1.0 \mathrm{~mm}$ )缠绕于收线轮, 轮内的卷簧保持一 定的预紧力, 当绳索出现松弛时, 按照图中方向将 松弛的绳索收紧, 使绳索保持张紧状态。可控离合 机构(图 $8 \mathrm{~b}$ )的功能是夹紧和释放绳索, 主要包括滚 珠自锁夹头、按压保持机构和推拉式电磁铁, 整个
机构高 $56 \mathrm{~mm}$, 长 $43 \mathrm{~mm}$, 宽 $20 \mathrm{~mm}$ 。

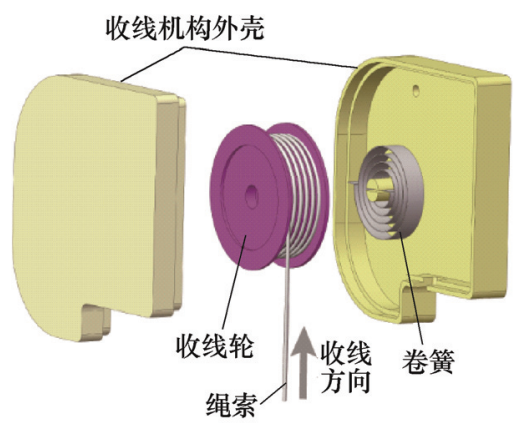

(a) 收线机构

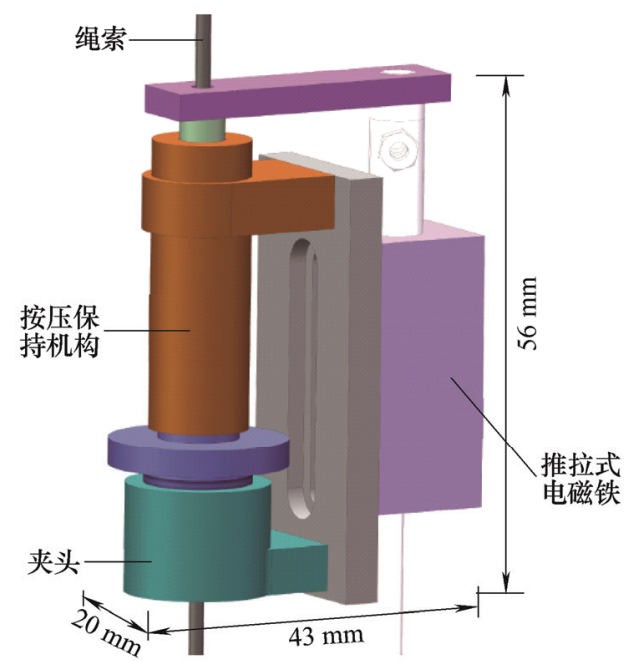

(b) 可控离合机构

图 8 串联弹性储能机构

\subsection{1 滚珠自锁夹头}

可控离合机构的夹头采用一种单向锁紧的滚珠 自锁装置作为夹持机构(图 9)。图 9 中绳索穿过钢珠 套筒, 3 个不锈钢珠(直径 $3.1 \mathrm{~mm}$ )置于套筒壁面的 孔中, 钢珠在夹头上半部壳体的圆锥内腔中运动。 钢珠套筒尾部的弹簧被夹头下半部挡住, 密封在壳 体的内腔中。由于弹簧对钢珠套筒始终保持推力, 钢珠与绳索保持挤压力。若向上拉动绳索则绳索与 钢珠间的摩擦力将带动钢珠一起向上运动, 此时钢 珠沿斜面运动更加向内挤压绳索, 形成自锁。当需 要松开绳索时, 只要向下按压钢珠套筒, 钢珠套筒 带动钢珠向后运动, 钢珠径向逐渐远离绳索从而将 绳索释放。适用于该夹头的绳索直径范围是 1 $2 \mathrm{~mm}$, 夹紧后的绳索最大可承受 $800 \mathrm{~N}$ 的拉力。本 研究选择推拉式电磁铁作为提供按压力的驱动装 置, 参数如表 3 所示。当需要松开绳索时, 电磁铁 通电推杆运动下压钢珠套筒松开绳索。 


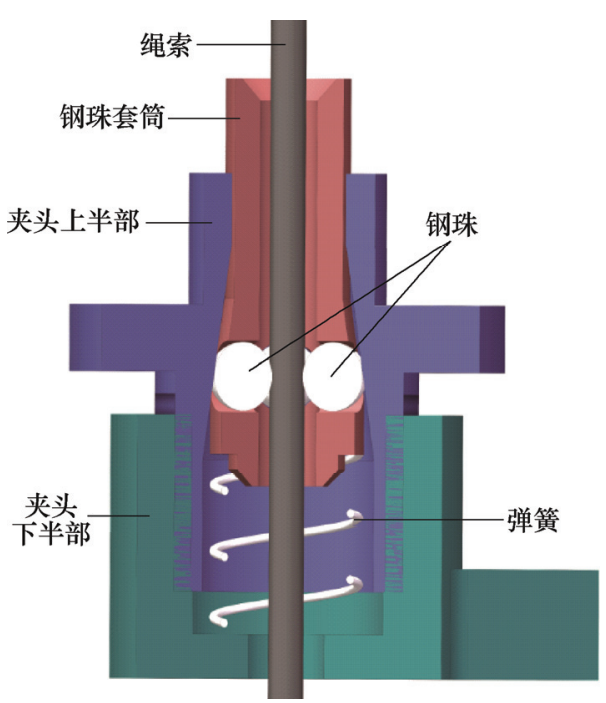

图 9 夹头机构

表 3 推拉式电磁铁参数

\begin{tabular}{cc}
\hline 参数 & 数值 \\
\hline 通电电压 $/ \mathrm{V}$ & 24 \\
按压行程 $/ \mathrm{mm}$ & 10 \\
推力 $/ \mathrm{N}$ & 5 \\
\hline
\end{tabular}

\subsection{2 按压保持机构}

由于上述绳索夹持机构在穿戴者行走时(如摆 动相期间) 需要保持相当长时间的释放状态, 为解决 电磁铁长时间通电容易烧毁的问题, 本研究受自动 圆珠笔棘轮按压机构的启发设计了一种按压保持机 构(图 10)。当上棘轮顶端被按下时, 下端的下棘轮 向下运动到一定位置后被棘轮套筒内的导向筋条卡 住, 阻止其再向上运动, 此时下棘轮停留在一个固 定位置(图 10 中的状态(1))。当再次按压时, 下棘轮 被解除限制并向上运动(图 10 中的状态(2)。根据这

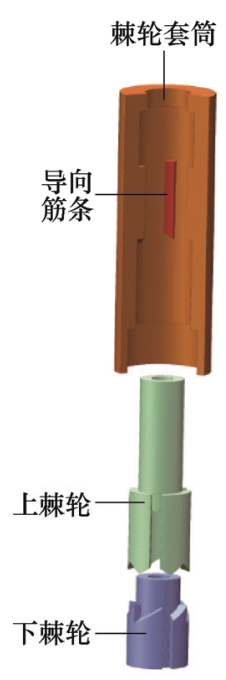

(1)按压保持状态

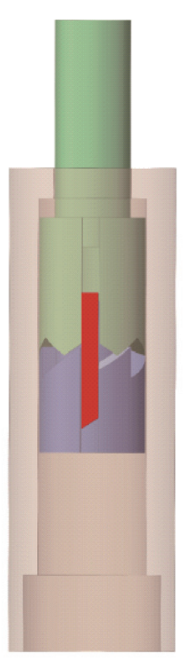

(2)解除限制状态
图 10 棘轮按压机构
种设计, 当需要释放绳索时, 只需要将电磁铁通电 施加按压力, 钢珠套筒便能保持在一个固定位置上, 此时即使电磁铁断电, 也没有影响, 大大减轻了电 磁铁长时间通电的负担。

下面分别对按压保持状态和解除限制状态之间 的切换过程进行受力分析, 根据受力情况选择合适 的设计参数。如图 11a 所示, 在上棘轮下压过程中, 下棘轮受到底端弹簧的推力 $F_{a}$ 以及上棘轮齿面对 其施加的正压力 $F_{n}$ 和摩擦 $f$, 并且沿着齿斜面方向 转动, 因此齿面摩擦力 $f$ 为滑动摩擦。根据受力平 衡条件可得

$$
\left\{\begin{array}{l}
f=\mu F_{n} \\
F_{a n}=F_{a} \cos \theta=F_{n} \\
F_{a t}=F_{a} \sin \theta>f
\end{array}\right.
$$

式中, $\mu$ 为齿面之间的摩擦系数, $\theta$ 为齿面坡度。 由方程组式(11)可知, 下棘轮沿齿斜面滑动的条件 为 $\mu<\tan \theta$ 。

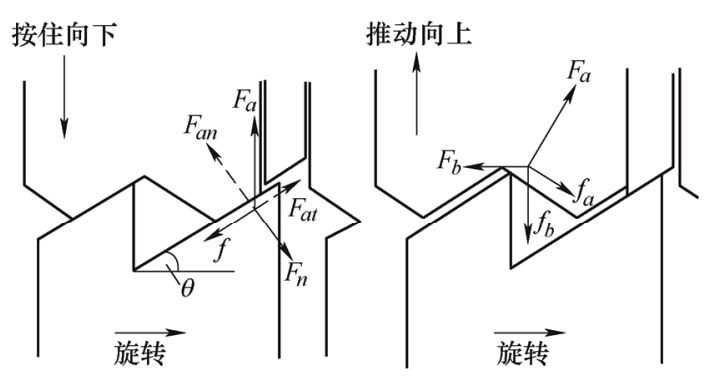

(a) 上棘轮下压过程

(b) 上柬轮回弹过程

图 11 状态切换时齿面受力分析

当上棘轮回弹时, 如图 $11 \mathrm{~b}$ 所示, 上棘轮受到 下棘轮沿齿斜面法向方向的推力 $F_{a}$ 以及由此产生 的摩擦力 $f_{a}$, 此外上棘轮还受到导向筋条施加的正 压力 $F_{b}$ 及摩擦力 $f_{b}$ 。根据其运动情况, 列出如下方 程组

$$
\left\{\begin{array}{l}
f_{b}=\mu F_{b} \\
f_{a}=\mu F_{a} \\
F_{b}=f_{a} \cos \theta+F_{b} \sin \theta \\
F_{a} \cos \theta>f_{b}+f_{a} \sin \theta
\end{array}\right.
$$

求解该方程组得 $\mu<-\tan \theta+\sqrt{1+(\tan \theta)^{2}}$ 。因此, 能够同时满足(a)和(b)两种运动状态的条件为

$$
\mu<\min \left\{\tan \theta,-\tan \theta+\sqrt{1+(\tan \theta)^{2}}\right\}
$$

当齿面坡度角满足式(13)时整个按压周期才能持 续, 否则就会产生摩擦角锁死的现象而无法正常 运转。

由于按压保持机构的结构较为复杂, 尤其是棘 
轮套筒内壁有多根导向筋条, 普通机床难以加工, 因此选择 3D 打印技术加工。为保证加工精度和零 件表面光滑, 选择光敏树脂作为 3D 打印的材料。 根据光敏树脂相同材料间的摩擦系数(约 0.2), 由式 (13)可确定齿面坡度角的范围为 $11.3^{\circ} \sim 67.4^{\circ}$, 本研 究选取的下棘轮齿面坡度角为 $30^{\circ}$ 。按压保持机构 的其他设计参数已在表 4 中列出。

表 4 按压保持机构设计参数

\begin{tabular}{cc||cc}
\hline 参数 & 数值 & 参数 & 数值 \\
\hline 套筒外径 $/ \mathrm{mm}$ & 10 & 齿面径向厚度 $/ \mathrm{mm}$ & 1 \\
套筒内径 $/ \mathrm{mm}$ & 7.2 & 下棘轮齿高 $/ \mathrm{mm}$ & 6 \\
套筒高度 $/ \mathrm{mm}$ & 31 & 上棘轮齿高 $/ \mathrm{mm}$ & 8.4 \\
导向筋条长度 $/ \mathrm{mm}$ & 8.8 & 上下棘轮中心孔直径 $/ \mathrm{mm}$ & 2.5 \\
\hline
\end{tabular}

\subsection{3 助力弹簧}

在人行走跖屈蹬地阶段, 用于助力的串联弹簧 和小腿处的跟腱近似平行, 其刚度系数的范围由支 撑相踝关节运动范围和足部弹性储能机构两侧弹簧 的拉力共同决定。为实现踝关节跖屈助力功能, 当 踝关节在支撑相并处于最大背屈状态时(图 12 中状 态(1)), 串联弹簧最大拉力 $F_{3}$ 应满足

$$
F_{3} D_{3}<F_{1} D_{2}
$$

式中, $D_{3}$ 为两侧板旋转轴到 $F_{3}$ 作用线的垂直距离。 根据人体测量学数据, 支撑相期间最大背屈角度约 $12^{\circ}{ }^{[6]}$, 此时 $D_{3}=167 \mathrm{~mm}, D_{2}=188.8 \mathrm{~mm}$, 两侧弹 簧总拉力 $F_{1}=248 \mathrm{~N}$ 时, $F_{3}$ 应小于 $280 \mathrm{~N}$ 。

考虑到串联弹簧的最大拉伸量为 $\Delta s=s_{2}-s_{1}=71 \mathrm{~mm}$, 其中, $s_{1}$ 为为最大背屈状态(图 12 中状态(1)时刻串联弹簧长度(约 $216 \mathrm{~mm}$ ), $s_{2}$ 为脚跟 着地后足底放平时刻(图 12 中状态(2)串联弹簧的初 始长度 $($ 约 $145 \mathrm{~mm}$ )。若假设弹簧的初拉力为零, 则 弹簧的刚度系数应小于 $3.9 \mathrm{~N} / \mathrm{mm}$ 。

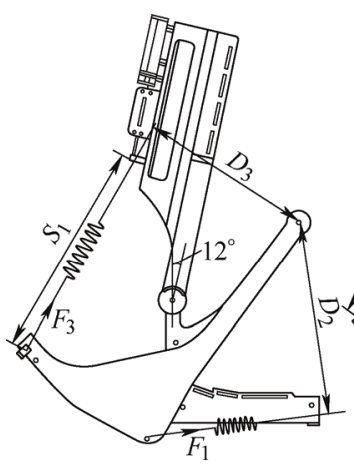

(1)最大背屈状态

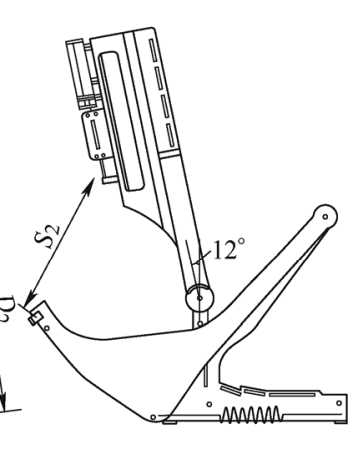

(2)足底放平状态
图 12 串联弹簧长度变化示意图

综合以上对外骨骼结构的描述，结合一个步态 周期内踝关节的运动特征对外骨骼的机构构件运动 过程描述如下(图 13)。

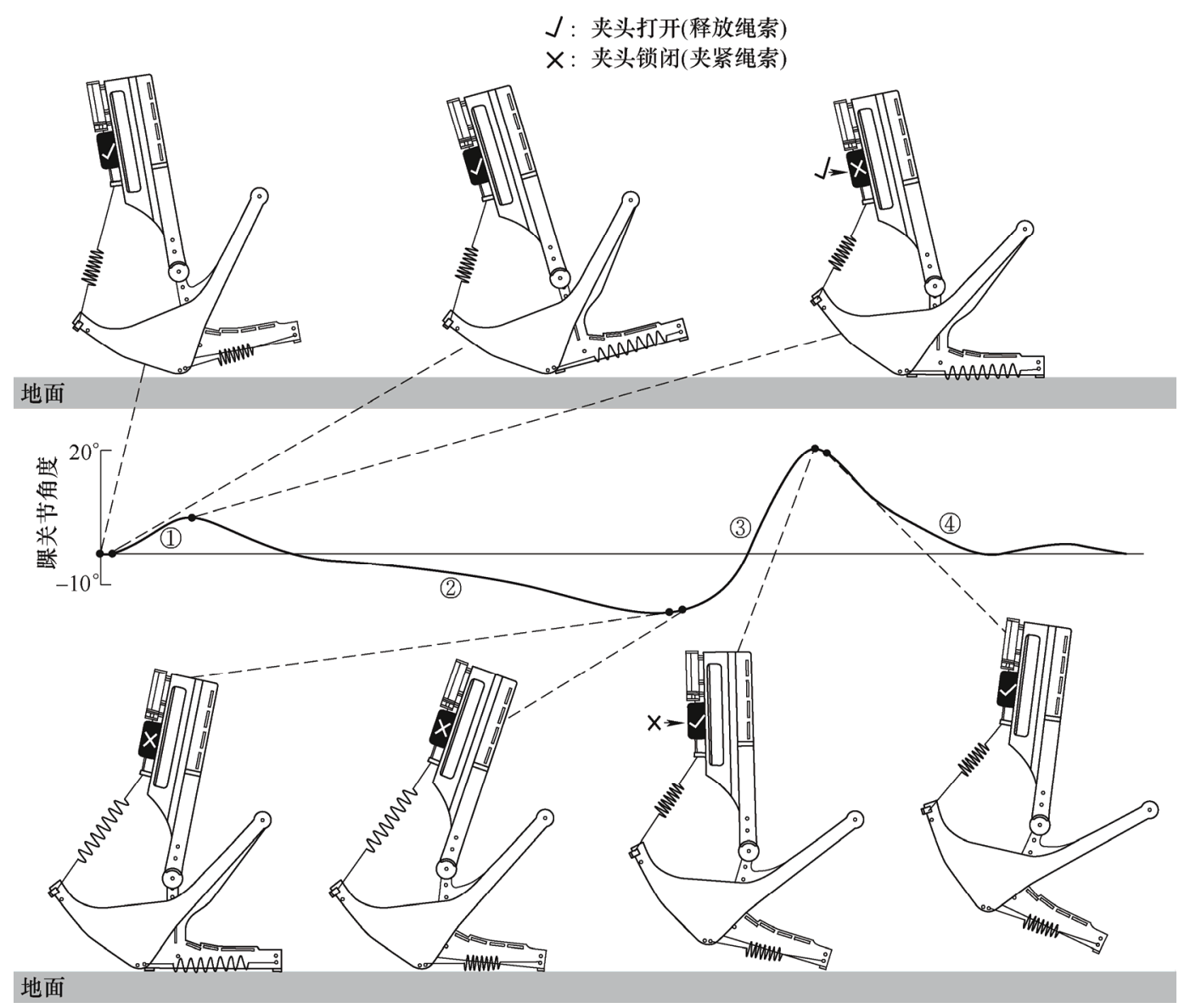

图 13 踝关节外骨骼工作原理 
（1）脚跟触地缓冲跖屈运动过程: 在摆动相即 将结束的时刻, 外骨骼足底储能机构的两侧板下边 缘早于人体脚跟先着地, 随着脚跟逐渐接近地面, 足底储能弹簧被拉伸( 储能), 直至脚底完全放平。 前脚掌与地面之间的压力信号超过一定阈值时, 触 发离合机构锁闭, 夹紧串联弹簧。

(2) 身体前移踝关节背屈运动过程: 从脚底放 平至支撑中相, 串联弹簧被拉伸(储能)。

(3) 蹬地前行踝关节跖屈运动过程: 在支撑末 期, 脚跟逐渐抬起, 足底储能弹簧逐渐收缩(释能), 将串联弹簧进一步拉伸(储能)。在蹬地阶段, 串联 弹簧收缩(释能)以助力踝关节跖屈蹬地。

(4) 摆动阶段: 在前脚掌与地面脱离过程中, 当接触力降低到一定阈值后, 触发离合机构释放串 联弹簧, 保证踝关节在摆动相期间自由转动, 防止 足尖擦地。

\section{4 踝关节外骨骼传感与控制系统}

根据上一节对外骨骼在一个步态周期内助力过 程的描述, 若要实现有效助力, 关键在于离合机构 的及时锁闭和打开, 该问题转化为准确反馈前脚掌 与地面的接触状态信号以控制电磁铁及时通断电, 从而驱动离合机构状态切换。本研究提出的踝关节 外骨骼的实时控制系统如图 14 所示, 该控制系统主 要硬件包括: STM32 开发板、继电器、足底压力传 感器(FlexiForce A301)、信号转换模块。

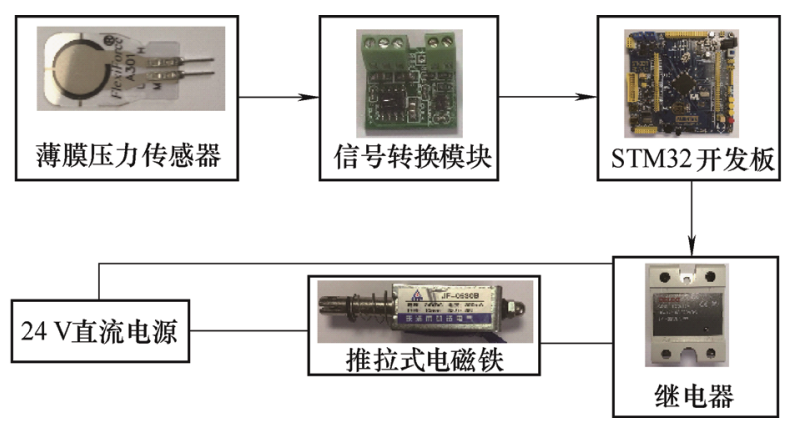

图 14 外骨骼的实时控制系统

本研究将薄膜压力传感器置于足底框架前脚板 的底部以检测前脚掌与地面的接触状态, 采集到的 压力信号如图 15 所示。当足底压力信号的电压值增 大(或减小)突破一定阈值时, 继电器导通, 电磁铁 两端接 $24 \mathrm{~V}$ 电压, 给按压保持机构施加按压力, 实 现离合机构的开闭切换, 其余时间继电器断路, 离 合机构保持闭合(或打开)状态。相较于文献[10]中依 赖于穿戴者行走时特定的踝关节角度触发离合机 构, 本外骨骼采用压力信号触发离合机构的状态切
换，可自动适应不同的穿戴者。

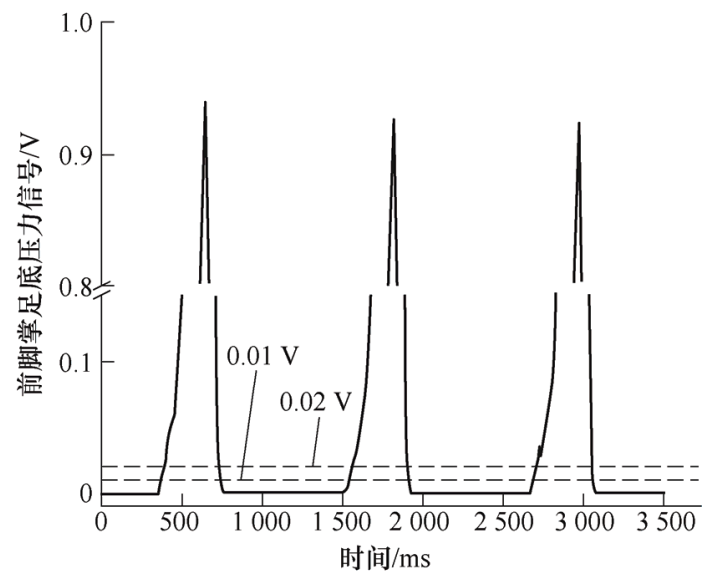

图 15 前脚掌压力信号(步速 $1.25 \mathrm{~m} / \mathrm{s}$ )

电磁铁触发流程如图 16 所示, 当前脚掌着地时 足底压力上升(图 16a), 在压力信号首次突破阈值 $0.02 \mathrm{~V}$ 后, 延迟 $10 \mathrm{~ms}$ 后再次采集, 若压力信号仍 大于 $0.02 \mathrm{~V}$, 则继电器导通, 电磁铁通电 $15 \mathrm{~ms}$, 否则, 认为是无效信号, 继续采集直到满足以上条 件。延迟 $10 \mathrm{~ms}$ 采集压力信号并再次与阈值比较的 目的是为了降低离合机构错误触发的概率。

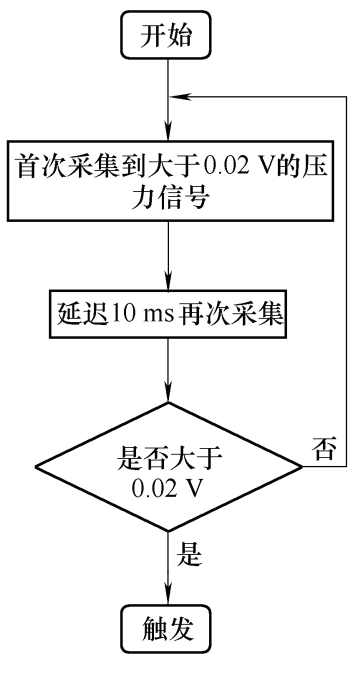

(a) 前脚掌压力增大

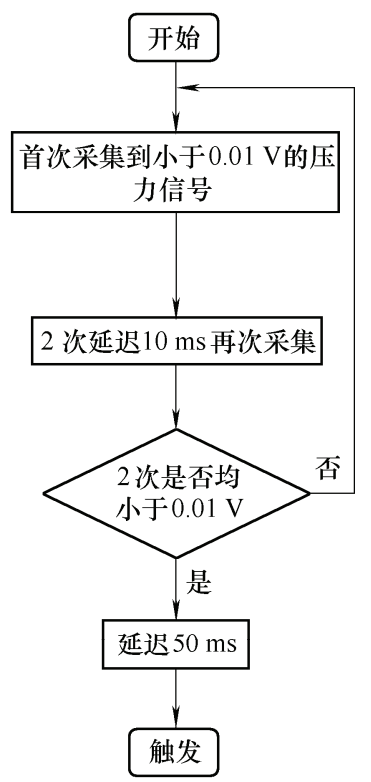

(b) 前脚掌压力减小
图 16 前脚掌压力信号控制的离合机构状态切换触发流程

当前脚掌离地时足底压力下降(图 16b), 在压力 信号首次跌破阈值 $0.01 \mathrm{~V}$ 后, 两次延迟 $10 \mathrm{~ms}$ 分别 采集压力信号, 若两次采集到的压力信号仍均小于 $0.01 \mathrm{~V}$, 则再延迟 $50 \mathrm{~ms}$ 发出继电器导通信号, 电 磁铁通电 $15 \mathrm{~ms}$ 。再延迟 $50 \mathrm{~ms}$ 的原因是当前脚掌足 底压力小于 $0.01 \mathrm{~V}$ 时, 串联弹簧有可能还存在一定 的拉力, 而根据夹持机构单向锁紧的特点, 当被夹 
紧的绳索有拉力时, 会导致夹头内部的钢珠和夹头 上半部的内壁存在较大静摩擦力(图 9), 若此时触发 继电器导通, 电磁铁输出的推力有可能无法克服该 静摩擦力, 导致离合机构状态切换失败。因此延迟 $50 \mathrm{~ms}$ 待串联弹簧拉力减为零以提高离合机构状态 切换成功的概率。

\section{5 样机试验}

为验证该踝关节外骨骼的可行性, 搭建的实验 平台如图 17 所示, 遴选一名健康受试者(男, 30 岁, 身高 $1.8 \mathrm{~m}$, 体重 $70 \mathrm{~kg}$ ) 在跑步机上进行实验验证。 本研究选用微型旋转增量式编码器 (型号: LQ_ECM141803_SDZ512)测量外骨骼关节的转动 角度和方向, 拉力传感器(型号: JLBS-M2-50KG, 量程为 $\pm 50 \mathrm{~kg}$ ) 测量串联弹簧的拉力。为验证外骨骼 助力效果, 选用肌电信号采集仪(Sichiray, 6 通道系 统, $1000 \mathrm{~Hz}$ )采集踝关节相关肌肉的肌电信号。由 于小腿三头肌(Triceps surae, TS)和胫骨前肌(Tibialis anterior，TA)这一对拮抗肌在人体行走时分别负责 踝关节在人体矢状面内的跖屈 ${ }^{[21]}$ 和背屈动作 ${ }^{[10]}$, 因 此本研究将这个两块肌肉作为研究对象, 采集其表 面肌电信号以评估肌肉激活度(图 18)。在采集表面 肌电信号时, 电极片黏贴在所要采集的肌肉腹部中 轴线附近, 电极片之间的间距大于 $3 \mathrm{~cm}$ 以避免电极 之间的串扰, 同时为保证每次测试都是相同的位置, 对电极片在皮肤上的位置进行了标注。

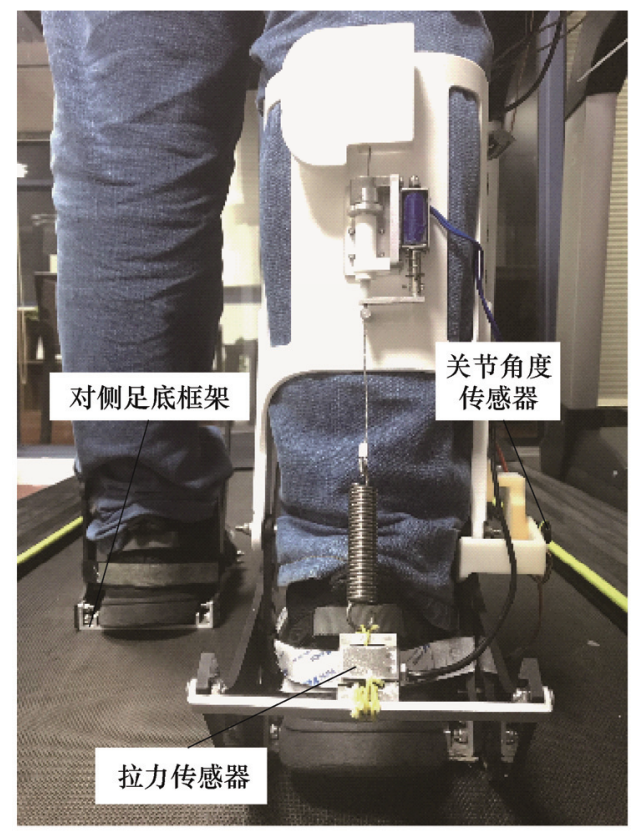

图 17 踝关节外骨骼样机平台

此外, 由于测试者仅在右腿穿戴了外骨骼, 而
外骨骼的足底框架会增加鞋底的厚度, 为了不影响 行走的对称性, 测试者的左脚也穿上了一个足底框 架, 确保行走时两条腿等长。在正式实验之前穿戴 者已经进行了约 $30 \mathrm{~min}$ 的适应性训练。

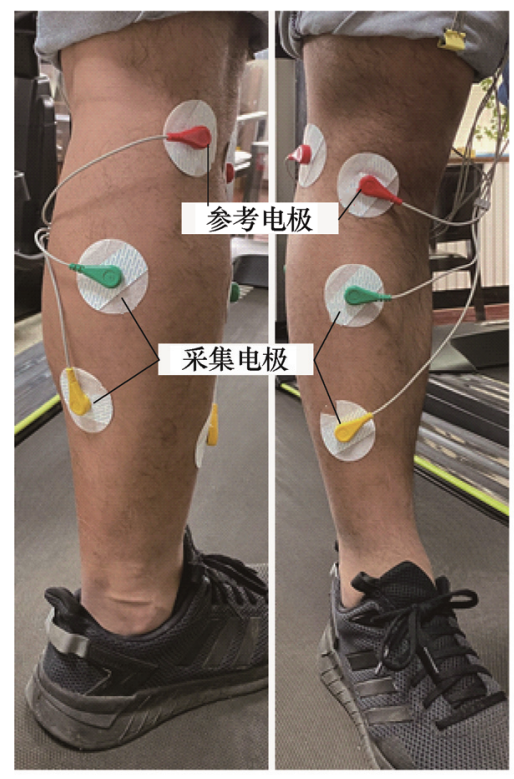

图 18 小腿三头肌和胫骨前肌表面肌电信号采集实验

\section{1 验证足底储能机构助力效果实验}

为验证足底储能机构的助力效果, 本研究采用 对比实验, 对比有与无足底储能机构两种条件下的 助力效果。首先, 采集测试者在不穿戴外骨骼( No Exo)以自然舒适的步速(约 $1.20 \mathrm{~m} / \mathrm{s}$ ) 行走时的踝关 节角度、小腿三头肌和胫骨前肌的表面肌电信号作 为比较的基准。然后, 穿戴完整外骨骼(Exo)进行行 走测试, 此时选取的串联弹簧刚度系数为 2.5 $\mathrm{N} / \mathrm{mm}$, 步速保持不变。最后, 将外骨骼足底弹簧去 掉(No Recycle), 两侧板分别用螺栓固定于足底框架 两侧进行行走测试, 串联弹簧刚度系数和行走步速 保持不变。Exo 和 No Recycle 测试中均采集外骨骼 踝关节角度值、小腿三头肌和胫骨前肌的表面肌电 信号以及串联弹簧拉力值。

采集到的原始肌电信号的处理方法如下: 首先 使用四阶巴特沃兹带通滤波器 $(20 \sim 400 \mathrm{~Hz}$ )进行滤 波处理, 以除去干扰噪声信号。然后将信号进行全 波整形和低通滤波(四阶巴特沃兹低通滤波器, $20 \mathrm{~Hz}$ ) 以提取其包络曲线 ${ }^{[22]}$ 。本研究采用表面肌电 信号的均方根(Root mean square, RMS)定量描述肌 肉激活度, 即计算一个步态周期内(从脚着地到同一 只脚下一次脚着地)目标肌肉群表面肌电信号包络 曲线的均方根 ${ }^{[23]}$ 。为保证肌电信号的稳定性, 选择 行走实验时最后连续 10 个步态周期的肌电信号, 分 别计算每个步态周期的均方根值。图 19 展示了 10 
个步态周期内小腿三头肌和胫骨前肌的原始信号和
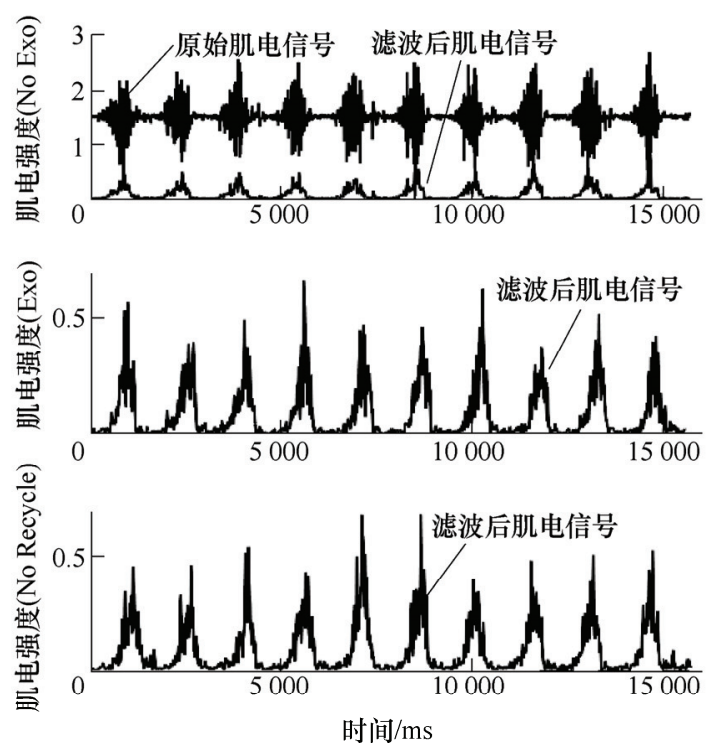

(a) 小腿三头肌
处理后的结果。
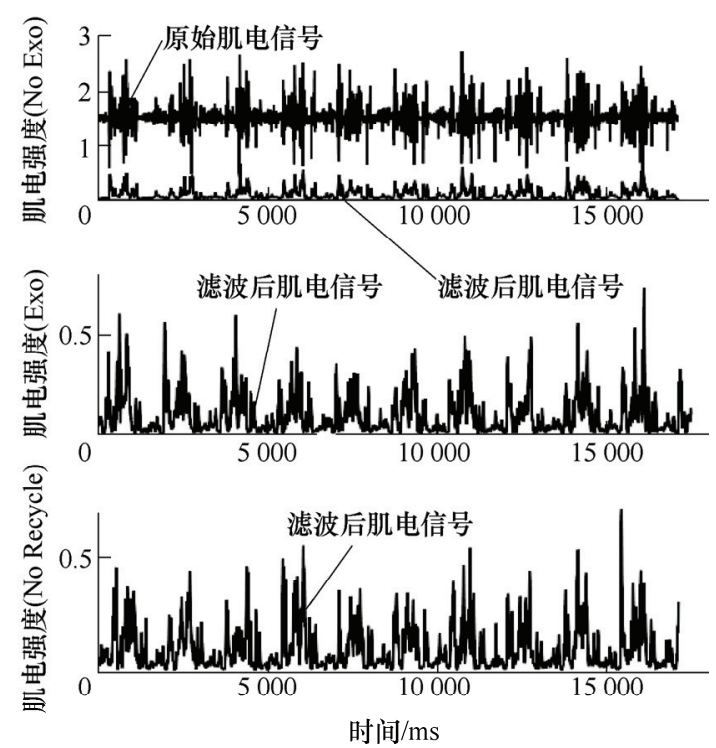

(b) 胫骨前肌

图 19 连续十个步态周期内, 不同行走条件时(No Exo、Exo、No Recycle)小腿三头肌和胫骨前肌表面肌电信号强度变化

定量比较足底储能机构的助力效果的方法如 下：首先，将不穿戴外骨骼(No Exo)自然行走时的 肌电信号进行上述处理得到 10 个步态周期的均方 根值, 将这 10 个值取平均值作为参考值。然后, 分 别计算 Exo 和 No Recycle 测试时最后连续 10 个步 态周期每个周期肌电信号的均方根值, 将这些均方 根值除以参考值进行归一化处理。最后, 计算这些 归一化值的平均值与标准差。以上操作对小腿三头 肌和胫骨前肌都适用。

图 20 展示了三种行走条件下(No Exo, Exo 和 No Recycle), 小腿三头肌和胫骨前肌肌电信号经上 述处理得到的平均值与标准差。由图可知, 在无足 底储能机构(No Recycle)时小腿三头肌的激活度相 较于自然行走(No Exo)下降约 4.8\%, 而引入该机构 后(Exo)进一步下降(约 7.2\%)。两种情况下胫骨前肌 的激活度相较于自然行走 (No Exo) 则略有上升 $(1.3 \%$ 和 $2.5 \%)$ 。根据小腿三头肌在人体行走时的工 作机理: 支撑腿踝关节背屈时, 小腿三头肌等长收 缩, 为跟腱的拉伸储能提供固着点 ${ }^{[24]}$ 。在支撑相末 期的蹬地时刻, 跟腱回弹释放储能, 同时小腿三头 肌向心收缩输出正功。在此过程中小腿三头肌通过 驱动踝关节跖屈蹬地提供了行走时超过 $50 \%$ 的机械 能 ${ }^{[25]}$ 。当穿戴外骨骼行走时, 由于可控夹头和串联 弹簧替代了小腿三头肌-跟腱系统的部分功能, 因此 减轻了小腿三头肌在踝关节背屈时的工作负担, 使 其激活度下降。而足底储能的释放又补偿了蹬地时 刻小腿三头肌输出的正功, 使其激活度进一步下降。
胫骨前肌激活度的轻微上升则是由于在支撑相早期 和中期 “对抗” 外骨骼力矩的结果 ${ }^{[10]}$ 。

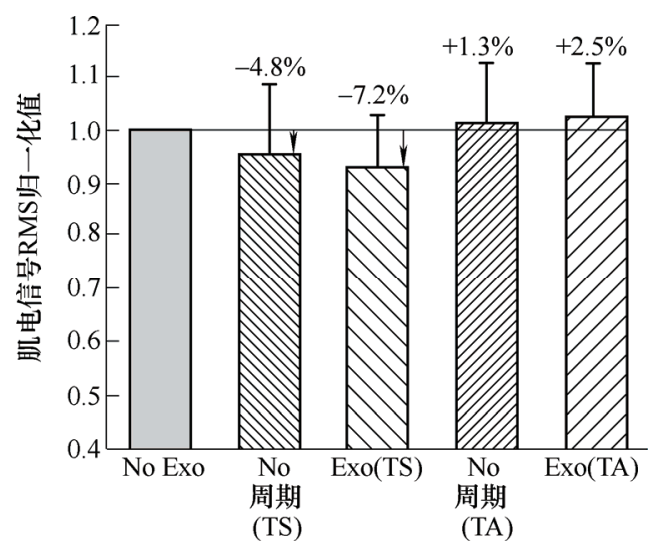

图 20 不同行走条件下(No Exo、Exo、No Recycle)小腿三

头肌(TS)与胫骨前肌(TA)肌电信号 RMS 归一化值比较

外骨骼的输出力矩等于串联弹簧的拉力值与外 骨骼旋转中心到串联弹簧拉力作用线垂直距离的乘 积。输出功率则为输出力矩与关节角速度的乘积。

图 21 分别展示了 Exo 和 No Recycle 两种条件下踝 关节角度、外骨骼输出力矩和功率在一个步态周期 内的变化曲线。由图可知, 添加足底储能弹簧后, 外骨骼输出力矩的峰值由 $8 \mathrm{~N} \cdot \mathrm{m}$ 增加到 $13 \mathrm{~N} \cdot \mathrm{m}$, 输 出功率的峰值由 $6 \mathrm{~W}$ 增加到 $17 \mathrm{~W}$ 。可见添加足底 储能机构提高了外骨骼的助力能力。

此外, 在两种穿戴情况下, 测试者在摆动相期 间( $60 \% \sim 100 \%$ 步态周期)的踝关节角度与 No Exo 时的踝关节角度相接近(相差在 $\pm 2^{\circ}$ 之间), 意味着在 
摆动相期间该踝关节外骨骼几乎没有干涉穿戴者的 踝关节活动 ${ }^{[10]}$ 。
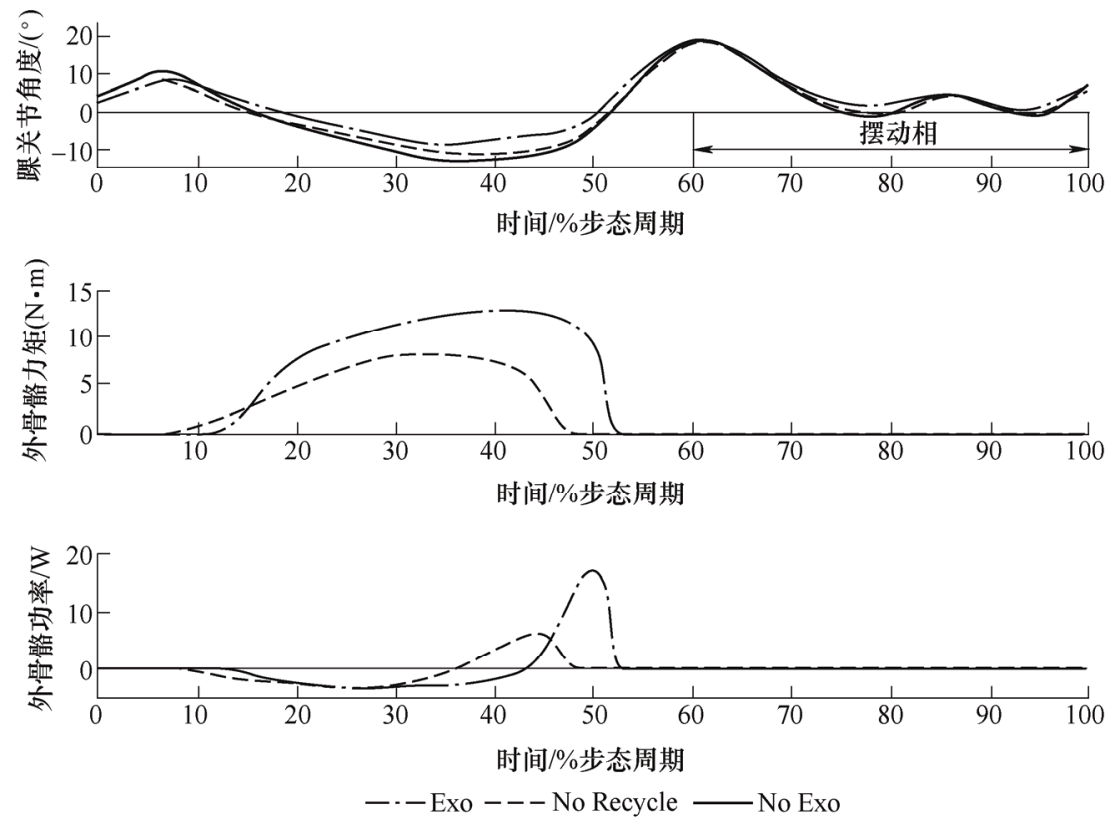

图 21 步态周期内踝关节角度、外骨骼力矩与功率

\section{2 串联弹簧不同刚度系数实验}

根据第 3.2.3 节中串联弹簧刚度系数范围(小于 $3.9 \mathrm{~N} / \mathrm{mm})$, 本研究选择刚度系数为 $1.5 \mathrm{~N} / \mathrm{mm}$ 、 $2.5 \mathrm{~N} / \mathrm{mm} 、 3.4 \mathrm{~N} / \mathrm{mm}$ 的弹簧分别开展助力行走实 验。穿戴者以同样的步速(约 $1.20 \mathrm{~m} / \mathrm{s}$ ) 行走, 采集三 次测试过程中踝关节角度、串联弹簧拉力、小腿三 头肌和胫骨前肌的表面肌电信号, 并根据第 5.1 节 的方法处理表面肌电信号并计算外骨骼的输出力矩 和功率。如图 22 所示, 随着串联弹簧刚度系数的增 加, 外骨骼输出力矩的峰值也随之增加 (分别为
$9 \mathrm{~N} \bullet \mathrm{m} 、 13 \mathrm{~N} \cdot \mathrm{m}$ 和 $19 \mathrm{~N} \cdot \mathrm{m})$, 最大峰值占到了人体 正常行走时踝关节最大关节力矩 $\left(\right.$ 约 $78 \mathrm{~N} \cdot \mathrm{m}^{[6]}$ ) 的 $24 \%$ 。当串联弹簧刚度系数为 $3.4 \mathrm{~N} / \mathrm{mm}$ 时, 外骨骼 的输出功率峰值约 $20 \mathrm{~W}$ 。随着串联弹簧刚度系数的 增加, 小腿三头肌的激活度呈下降趋势(最大下降约 $8.6 \%$ ), 胫骨前肌的激活度呈上升趋势(最大上升约 $3.2 \%$ ), 如图 23 所示。值得注意的是, 随着串联弹 簧刚度系数的增加, 人体行走的能耗并非一直减小, 而是呈现 “ $U$ ” 形 ${ }^{[10]}$ 。
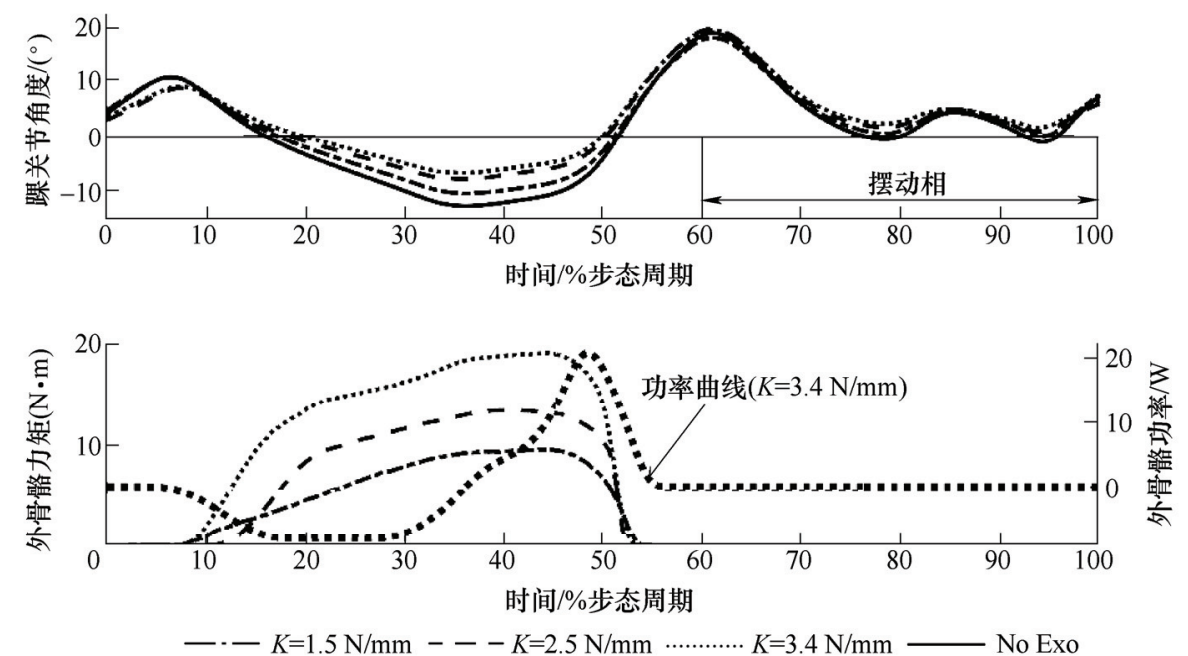

图 22 步态周期内踝关节角度、外骨骼输出力矩与功率

此外，三种情况下，在摆动相期间(60\% 100\% 步态周期)外骨骼踝关节的角度与 No Exo 时的踝关
节角度相差在 $-1^{\circ} \sim+2.5^{\circ}$ 之间(图 22 ), 意味着外骨骼 在摆动相期间很少干涉踝关节的正常活动 ${ }^{[10]}$ 。 


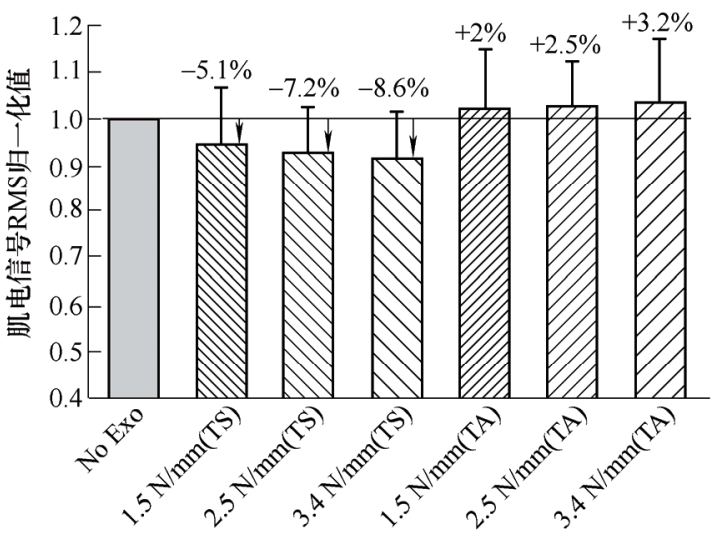

图 23 串联弹簧不同刚度系数条件下小腿三头肌(TS)与胫 骨前肌(TA)肌电信号 RMS 归一化值比较

\section{6 结论}

人体行走是神经系统和肌肉-骨骼系统协同合 作的产物, 具有高度复杂性和低能耗的特点。人体 行走的复杂性导致研究者不易探究行走过程中的能 耗规律。本文提出了一种基于耦合摆模型分析人体 行走的方法, 并建立人体行走摆动相动力学模型, 采用打靶法求解摆动相期间各关节的角度变化曲 线。计算结果表明, 各关节角度的理论值接近实测 值。然后计算得到 Heel-strike、Knee-lock 及关节摩 擦引起的能耗率。结果表明, Heel-strike 的能耗率 远大于 Knee-lock 和关节摩擦的能耗率。

基于以上能耗分析, 本研究提出了一种有源无 动力踝关节外骨骼, 所耗电能主要用于离合机构的 驱动和传感与控制系统。该外骨骼利用足底弹性储 能机构将摆动腿 Heel-strike 的能耗存储起来, 在蹬 地时刻释放实现助力行走。采集前脚掌压力信号控 制电磁铁驱动的离合机构, 实现串联弹簧的夹紧与 释放状态切换。为验证外骨骼的可行性, 分别进行 了有无足底储能弹簧的对比实验和采用不同刚度系 数串联弹簧的对比实验。样机实验结果表明: 足底 储能机构可提高外骨骼的输出助力力矩和功率, 穿 戴该外骨骼行走可以降低小腿三头肌的激活度(胫 骨前肌的激活度则略有上升)。此外, 关节角度的测 量结果表明, 该外骨骼在摆动相期间很少干涉穿戴 者踝关节的正常活动。

未来需要进一步开展的工作包括: (1) 以轻型化 为目标, 对现有的外骨骼机构进行拓扑优化设计;

(2) 开展样机户外行走研究, 探究在高低不平的户外 采用其他传感器(如微型惯性传感器)检测步态的相 位信息, 进行基于步态相位识别的离合器控制策略 研究, 拓宽该外骨骼的适用范围; (3) 开展双足外骨
骼效能评价研究, 采用测量耗氧量计算新陈代谢率 的方法来评价其效能。

\section{参 考 文 献}

[1] TUDOR-LOCKE C, JOHNSON W D, KATZMARZYK $\mathrm{P}$ T. Accelerometer-determined steps per day in US adults[J]. Medicine \& Science in Sports \& Exercise, 2009, 41(7): 1384-1391.

[2] CHOI Y, KIM Y, KIM M, et al. Muscle synergies for turning during human walking[J]. Journal of Motor Behavior, 2019, 51(1): 1-9.

[3] JIANG S, WANG Y, ZHANG Y, et al. Real time gait recognition system based on kinect skeleton feature[M]. Springer International Publishing, 2014.

[4] CAPADAY C. The special nature of human walking and its neural control[J]. Trends in Neurosciences, 2002, 25(7): $370-376$.

[5] O'CONNOR S M, DONELAN J M. Fast visual prediction and slow optimization of preferred walking speed[J]. Journal of Neurophysiology，2012， 107(9): 2549-2559.

[6] 韩亚丽, 贾山, 王兴松. 基于人体生物力学的低功耗踝 关节假肢的设计与仿真 $[\mathrm{J}]$. 机器人, 2013, 35(3): 276-282.

HAN Yali, JIA Shan, WANG Xingsong. Design and simulation of an ankle prosthesis with lower power based on human biomechanics[J]. ROBOT, 2013, 35(3): 276-282.

[7] FARRIS D J, SAWICKI G S. The mechanics and energetics of human walking and running: a joint level perspective[J]. Journal of the Royal Society Interface, 2012, 9(66): 110-118.

[8] MOONEY L M, ROUSE E J, HERR H M, et al. Autonomous exoskeleton reduces metabolic cost of human walking[J]. Journal of Neuroengineering and Rehabilitation, 2014, 11(1): 1-6.

[9] ZHANG J , FIERS P, WITTE $\mathrm{K}$ A, et al. Human-in-the-loop optimization of exoskeleton assistance during walking[J]. Science, 2017, 356(6344): 1280-1284.

[10] COLLins S H, WIGGIN M B, SAWICKI G S, et al. Reducing the energy cost of human walking using an unpowered exoskeleton[J]. Nature, 2015, 522(7555): 212-215.

[11] YANDELL M B, TACCA J R, ZELIK K E. Design of a low profile, unpowered ankle exoskeleton that fits under clothes: Overcoming practical barriers to widespread societal adoption[J]. IEEE Transactions on Neural 
Systems and Rehabilitation Engineering, 2019, 27(4): 712-723.

[12] DILLER S, MAJIDI C, COLLINS S H. A lightweight, low-power electroadhesive clutch and spring for exoskeleton actuation[C]// Proceedings of the 2016 IEEE International Conference on Robotics and Automation (ICRA), May 16-21, 2016, Stockholm, Sweden, 2016: 682-689.

[13] WANG X, GUO S, QU H, et al. Design of a purely mechanical sensor-controller integrated system for walking assistance on an ankle-foot exoskeleton[J]. Sensors, 2019, 19(14): 3196.

[14] 任孟沂, 曹恩国, 赵永武, 等. 一种被动式外骨骼机械 足的结构设计及优化 [J]. 工程设计学报, 2020, 27(2): 199-211.

REN Mengyi, CAO Enguo, ZHAO Yongwu, et al. Design and optimization of a passive exoskeleton mechanical foot[J]. Chinese Journal of Engineering Design, 2020, 27(2): 199-211.

[15] WANG T M, PEI X, HOU T G, et al. An untethered cable-driven ankle exoskeleton with plantarflexiondorsiflexion bidirectional movement assistance[J]. Frontiers of Information Technology \& Electronic Engineering, 2020, 21(5): 723-739.

[16] CHANG Y, WANG W, FU C. A lower limb exoskeleton recycling energy from knee and ankle joints to assist push-off[J]. Journal of Mechanisms and Robotics, 2020, 12(5): $1-17$.

[17] SAWICKI G S, BECK O N, KANG I, et al. The exoskeleton expansion: improving walking and running economy[J]. Journal of Neuro Engineering and Rehabilitation, 2020, 17(1): 17-25.

[18] SRINIVASAN M, RUINA A. Computer optimization of a minimal biped model discovers walking and running $[\mathrm{J}]$. Nature, 2006, 439(7072): 72-75.

[19] LEVEAU B F, WILLIAMS M. Williams \& Lissner's biomechanics of human motion[M]. W.B. Saunders Co., 1992.

[20] ISHIKAWA M, KOMI P V, GREY M J, et al. Muscle-tendon interaction and elastic energy usage in human walking[J]. Journal of Applied Physiology，2005, 99(2): 603-608.

[21] FHONEINE J L, SCHIEPPATI M, GAGEY O, et al. The functional role of the triceps surae muscle during human locomotion[J]. Plos One, 2013, 8(1): e52943.

[22] DING Y, GALIANA I, ASBECK A, et al. Biomechanical and physiological evaluation of multi-joint assistance with soft exosuits[J]. IEEE Transactions on Neural Systems and Rehabilitation Engineering, 2017, 25(2): 119-130.

[23] YONG J R, SILDER A, DELP S L. Differences in muscle activity between natural forefoot and rearfoot strikers during running $[\mathrm{J}]$. Journal of Biomechanics, 2014, 47(15): 3593-3597.

[24] ZELIK K E, HUANG T, ADAMCZYK P G, et al. The role of series ankle elasticity in bipedal walking[J]. Journal of Theoretical Biology, 2014, 346: 75-85.

[25] FARRIS D J, SAWICKI G S. Human medial gastrocnemius force-velocity behavior shifts with locomotion speed and gait[J]. Proceedings of the National Academy of Sciences, 2012, 109(3): 977-982.

作者简介: 王存金, 男, 1988 年出生, 博士研究生。主要研究方向为生 物力学、下肢外骨骼运动学、动力学及控制。

E-mail: jiajia1900s@163.com

王兴松(通信作者), 男, 1965 年出生, 博士, 教授。主要研究方向为机 器人动力学及其控制、生物机械电子、先进医疗器械等。

E-mail: xswang@seu.edu.cn 